\section{Three dimensions to theory in chemistry}

\section{Jennifer Green}

Orbital Interactions in Chemistry. By T.A. Albright, J.K. Burdett and M.H. Whangbo. Wiley: 1985. Pp.447. \$49.95, $£ 51.10$.

Chemists are very receptive to pictorial representations of their science, and are well-used to thinking in three dimensions. Albright, Burdett and Whangbo take full advantage of this ability in their book, which contains a myriad of graphic representations of orbital interactions in molecules. Their aim is to describe and examine the electronic structure of covalent molecules and solids with a view to understanding their existence, structure and, in some cases, their reaction pathways. The strength of the semi-empirical approach on which such orbital descriptions are based, and the "cartoons" in which the results are communicated, is their accessibility to the non-mathematical chemist, who is thereby provided with a set of theoretical tools for prediction, as well as rationalization, of structure and reactivity.

In a carefully planned first section the quantum mechanical and group theoretical basis of this semi-empirical approach is clearly expounded. Within a framework of LCAO-MO theory, symmetry, overlap, electronegativity and perturbation arguments are used to establish the orbital structure of simple cases. Here many motifs are outlined which recur, with elaborations, throughout the book. The implications of various occupancies of these prototype orbital structures are discussed; exchange energy, first and second JahnTeller effects, the Fock operator and configuration interaction are brought into play.

The second section consists of an exploration of main group covalent molecules using the technique of building up the orbital structure of larger molecules from that of smaller fragments, while maintaining a delocalized picture of the orbitals. Where relevant, the relation to a localized bond picture is made clear. The third section is a similar treatment of transition metal complexes. The extensive range of molecules discussed, from the simplest organics to metal clusters, bears witness to the power of the technique.

\section{New editions}

- The Meaning of Fossils: Episodes in the History of Palaeontology, 2nd Edn, by Martin J.S. Rudwick. Publisher is University of Chicago Press, price is pbk $\$ 11.95, £ 11.25$.

- Flame and Combustion, 2nd Edn, by J.A. Barnard and J.N. Bradley. Publisher is Chapman and Hall, price is pbk $112.95, \$ 27$; hbk $£ 25, \$ 55$.
BOOK REVIEWS

However, as the density of examples increases the clarity of the explanations declines. The emphasis on coverage, rather than exemplification, makes the text less accessible than it might have been to the undergraduate, but more useful to research chemists who will appreciate having this large body of work brought together within a single volume.

Throughout, the unity of approach is impressive for a book written by three authors, and can be attributed in part to the influence, acknowledged in the preface, of Roald Hoffmann (who also contributed the foreword). The style of writing is less even but at all points the illustrations help in understanding the text and vice versa. Particularly welcome is a chapter on solids, which attempts the Herculean task of translating the language of solid state physics for the benefit of the chemist with only a moderate acquaintance with molecular orbital theory. For this to be entirely successful a complete book would be needed, but this short account is very helpful.

The book gives a rather bland impression of the periodic table; perhaps the blend of overlap and symmetry arguments could have been seasoned by more emphasis on the periodic trends of orbital energies and separations, which contribute to the uniqueness of each element's chemistry. However, as a compilation and clear exposition of the work of an influential school of theoretical chemistry it will be extremely useful.

Jennifer (jreen is a Fellow' of St Hugh 's College. Oxford OX2 6LE. UK.

\section{A wealth of viruses}

\section{D.J. McCance}

Virology. Edited by Bernard N. Fields. Raven:1985. Pp.1,614. \$176.50.

Aт 4.75 kilograms there is no disputing the publisher's intention that this tome should be a reference book; no one would consider carrying it around for light reading. With the title Virology one would expect a broadly based text and indeed this is the case, except that only mammalian viruses of medical importance are discussed (plant viruses are relied on heavily in the sections on viral structure however).

This is the first attempt to cover mammalian virology comprehensively since 1974 when Fenner et al.'s The Biology of Animal Viruses appeared, and the increase in size over that book reflects the advances of the past ten years in our knowledge of virus structure, genetics, replication and pathogenesis. The dangers of a volume such as this, of course, are that with so many contributors (here 64) it takes time to come to press and because of constraints on space the authors may be tempted into superficiality. Both pitfalls have been avoided: the various chapters are rich in detail and, judging by the dates of papers in the reference lists, the editor managed to cajole his authors into meeting their deadlines.

There are really two parts to the book, the first third of it dealing with the general principles of medical virology and the rest with individual viruses or virus groups. In this second part, the molecular biology and pathogenesis of particular viruses are discussed in successive chapters, thus providing easy access to information of interest to people of different backgrounds. With this format the book will be useful to a wide range of individuals, including undergraduates from related disciplines, molecular virologists and clinicians. The clinician should take comfort in the fact that in general the molecular virology aspects are well illustrated with informative and digestible figures to help in comprehending the complexities of virus structure and replication.

The wide-ranging nature of the book gives the reader an interesting perspective on the state of virology during the past decade, which has seen the discovery, for example, of the human retroviruses, HTLV I-III, the rotaviruses causing gastroenteritis and the hepatitis viruses. It is also possible to discern the renewed emphasis placed on previously identified virus groups, often because of the inroads made through molecular biological techniques. A decade ago the pathogenesis of the genus papillomavirus would have only been mentioned. Now, given their association with some genital malignancies, they command half the chapter on the Papovaviridae, while hepatitis A and B, non- $A$, non-B and the Delta agent take three chapters. Unfortunately genetic engineering has not been as productive in other areas, such as viral chemotherapy, where progress has been much slower.

Overall the book succeeds in covering medical virology in depth, and with its helpful illustrations and extensive bibliographies will find wide use as a reference text. It is clear that although great progress has been made on the molecular side of the subject, our knowledge of the process of pathogenesis lags well behind. One can hope that the book's structure will encourage those from either molecular or clinical backgrounds to browse through both types of chapter, and result in more people becoming interested in pathogenesis at the molecular level. Such individuals are needed to utilize molecular techniques in investigating the pathogenesis of virus diseases and to produce effective treatment and preventive measures. ment of Microbiology, United Medical and Dental Schools of Guy's and St Thomas's Hospitals, Guy's Campus, London Bridge, London SEl $9 R T$, UK.
D.J. McCance is Senior Lecturer in the Depart- 